\title{
Bioavailability in Man of Iron in Human Milk and Cow's Milk in Relation to Their Calcium Contents
}

\author{
LEIF HALLBERG, LENA ROSSANDER-HULTÉN, MATS BRUNE, AND ANN GLEERUP \\ Department of Medicine II and Department of Clinical Nutrition, University of Göteborg, Sahlgren Hospital,
} S-41345 Göteborg, Sweden

\begin{abstract}
Iron absorption from human milk and cow's milk was compared in the same subjects using two radioiron tracers and extrinsic labeling of the iron. Previously observed higher iron absorption from human milk was confirmed as $19.5 \pm 17.3 \%$ (mean $\pm \mathrm{SD}$ ) in cow's milk versus $48.0 \pm 25.5 \%$ in human milk; mean individual absorption ratio was $0.39 \pm 0.18(p<0.0001)$. Cow's milk has a several times higher content of calcium, recently established to be a potent inhibitor of iron absorption. Adding calcium chloride to human milk in amounts to equate the calcium content of cow's milk significantly reduced iron absorption $[39.1 \pm 17.8 \%$ (mean \pm SD) versus $21.3 \pm 10.6 \%$; mean individual absorption ratio with or without added calcium was $0.58 \pm 0.12$ (mean \pm SD) $(p<$ 0.0001). Differences in calcium content could explain at least $70 \%$ of the difference in iron bioavailability between milks. It is suggested that the remaining $\mathbf{3 0} \%$ difference may mainly be an artifact related to inhomogeneous radioiron-labeling of milk iron compounds, especially in human milk, and that the main difference in true iron absorption between human and cow's milk is related to their different calcium contents. The rather high fractional iron absorption from human milk can be explained by its low iron content. There is nothing to indicate that human milk contains any enhancer of iron absorption. The marked inhibiting effect of calcium on iron absorption should be considered in the design of infant formulas to achieve an optimal balance in the contents of calcium and iron. (Pediatr Res 31: 524-527, 1992)
\end{abstract}

Previous studies strongly suggest that iron absorption from human milk is considerably higher than that from cow's milk (1). This is valid for both the fractional and absolute amount of iron absorbed. In the only comparative study published, the iron absorption from human milk and cow's milk was 20.8 and $13.6 \%$, respectively (2). The study included 10 adults. In another study made by the same group on five adult men, the average iron absorption from human milk was in the same range, $23.7 \%$ (3). In a study on 11 infants (4), 5.9 to $7.3 \mathrm{mo}$ of age, the average iron absorption from human milk was $48.8 \pm 26.2 \%$ (mean \pm SD). Differences in iron status between adults and infants is a probable reason for these differences in iron absorption from human milk. All these studies were made by extrinsic labeling of

Received September 12, 1991; accepted November 28, 1991.

Correspondence and reprint requests: Leif Hallberg, Department of Medicine II, University of Göteborg, Sahlgren Hospital, S-413 45 Göteborg, Sweden.

Supported by Swedish Medical Research Council Project B88-19X-04721-13B, Swedish Council for Forestry and Agriculture Research 864/86, 31:2, and 59989L-1 35:2 and Swedish Agency for Research Co-operation with Developing Countries $9.49 /$ SAREC 85/46:2. the milk iron. In one early, classic study, the absorption of iron was measured from biosynthetically labeled cow's milk produced by giving large doses of radioiron to a lactating cow (5). In 10 children aged 4 to $52 \mathrm{mo}$, most iron absorption values were within the range $10-15 \%$; in four iron-deficient infants, 11 to 18 mo of age, the absorption was slightly higher, $11-24 \%$. It may thus be summarized from the previous studies that iron absorption from human milk is higher than that from cow's milk and that the iron absorption from cow's milk is about $10-15 \%$. The cause of the observed difference in bioavailability of iron in human milk and cow's milk has not been established, but several differences in chemical composition have been suggested as explanations (for a review, see Ref. 1).

Recently, it has been shown that the calcium in a meal markedly reduces, in a dose-related manner, the bioavailability of iron (6). Inasmuch as cow's milk contains about four times more calcium than human milk, this may be an explanation for the difference in bioavailability of iron. The purpose of this study was to examine the effect on iron absorption of equating the calcium content in human milk to that in cow's milk by adding a calcium salt. Iron absorption from human milk with and without extra calcium was compared in the same subjects using two different radioiron isotopes to reduce the marked confounding effect of intersubject variation in iron absorption.

\section{MATERIALS AND METHODS}

Subjects. Altogether, 18 healthy volunteers participated in the two studies: five men and 13 women between 19 and 39 y of age. Some of the subjects were regular blood donors, so a reasonable range of intersubject variation in iron absorption was ensured (Table 1). Participating subjects were given oral and written information about the aims and procedures of the study. The study was approved by the Ethical Committee of the Medical Faculty of the University of Göteborg, Sweden.

Experimental design and measurement of iron absorption. In one study (nine subjects), a comparison was made between the absorption of iron from human milk and cow's milk. Comparisons were made in the same subject by labeling the milk iron with two different radioiron isotopes, ${ }^{55} \mathrm{Fe}$ and ${ }^{59} \mathrm{Fe}$. In the other study (nine subjects), iron absorption was measured from human milk given with or without extra calcium to equate the higher calcium content in cow's milk. The calcium added was calcium chloride.

In each study, the two differently radioiron-labeled "meals" (milk samples) to be compared (A and B) were served on four consecutive mornings after an overnight fast in the order ABBA or BAAB. No food or drink was allowed for $3 \mathrm{~h}$ after the meal. Each meal consisted of $200 \mathrm{~mL}$ of milk. Two wk after the last serving, a fasting blood sample was drawn to determine the content of ${ }^{59} \mathrm{Fe}$ and ${ }^{55} \mathrm{Fe}$ in circulating blood. At the same time, the total retention of ${ }^{59} \mathrm{Fe}$ was measured by whole-body counting. 
Table 1. Iron absorption from human milk and cow's milk (study 1) and human milk without and with addition of 160 mg calcium to equate calcium content in human milk and cow's milk (study 2)*

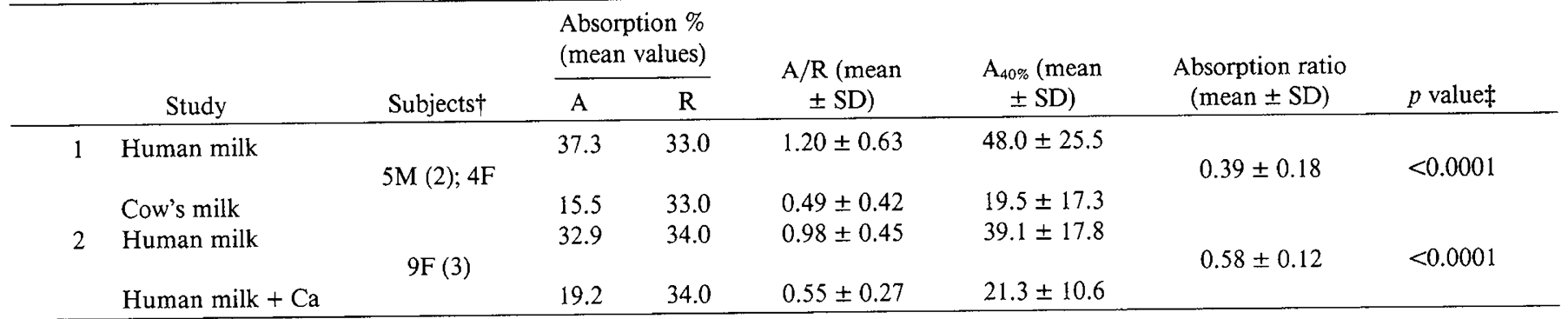

* $A$, absorption of iron from milk; $R$, absorption of iron from reference doses; mean value from $A$ at $A_{40 \%}$, mean value from $A$ at $40 \%$ referencedose absorption.

$\dagger$ Number of subjects. M, male subjects; $F$, female subjects. Number of blood donors in parentheses.

$\ddagger p$ values represent the probability that the absorption ratio values are different from 1 .

The total retention of ${ }^{55} \mathrm{Fe}$ was calculated from the ratio of ${ }^{55} \mathrm{Fe}$ and ${ }^{59} \mathrm{Fe}$ in red cells.

To account for the greatly varying iron absorption in different subjects, a reference dose of inorganic iron labeled with ${ }^{59} \mathrm{Fe}$ was then given (see below), and a second dose was given on the morning after an overnight fast. The iron absorption from the reference doses was then measured by whole-body counting 2 wk later (see the expression of results below).

The radioiron as high $\mathrm{sp}$ act ferric chloride in $0.01 \mathrm{M}$ hydrochloric acid was mixed into the milk before serving. Each portion of milk was labeled with $46-\mathrm{kBq}{ }^{59} \mathrm{Fe}$ or $55-\mathrm{kBq}{ }^{55} \mathrm{Fe}$.

Iron absorption measurements. From analyses of the contents of ${ }^{55} \mathrm{Fe}$ and ${ }^{59} \mathrm{Fe}$ in the blood samples drawn 2 wk after the last serving, the relative absorption of the two isotopes was calculated, assuming a certain fraction of absorbed iron present in red cells and a certain blood volume in relation to body weight. Absolute absorption of ${ }^{59} \mathrm{Fe}$ was calculated from the retention of ${ }^{59} \mathrm{Fe}$ measured by whole-body counting. Absolute absorption of ${ }^{55} \mathrm{Fe}$, which cannot be measured by whole-body counting, was calculated from the relative absorption of the two tracers and the absolute absorption of ${ }^{59} \mathrm{Fe}$. Analysis of ${ }^{55} \mathrm{Fe}$ and ${ }^{59} \mathrm{Fe}$ in blood was made by a modification of the method described by Eakins and Brown (7). All procedures and methods of calculation have been described previously $(8,9)$.

Oral reference doses. A solution of $10 \mathrm{~mL}$ of $0.01 \mathrm{~mol}$ hydrochloric acid/L containing $3 \mathrm{mg}$ of $\mathrm{Fe}$ as $\mathrm{FeSO}_{4}$ and $30 \mathrm{mg}$ of ascorbic acid labeled with ${ }^{59} \mathrm{Fe}$ was used as a reference in all studies. The $10-\mathrm{mL}$ vials containing the Fe solution were rinsed twice with water, and this liquid was also consumed. Each subject received two reference doses on two consecutive mornings after an overnight fast. No food or drink was allowed for $3 \mathrm{~h}$ after the reference dose. Each subject received a total of $18 \mathrm{kBq}$ of ${ }^{59} \mathrm{Fe}$ in the two reference doses. The absorption from the reference doses was measured by a new whole-body count 2 wk later.

Expressing results of absorption measurements and statistical analyses. In each subject, the ratio (A:R) of absorption of iron from a milk meal (A) and from reference doses (R) is an expression of the bioavailability of nonheme Fe in the milk. In a selected group of subjects, these ratio values can be considered to be normally distributed, and the mean and SD of the ratio values are thus calculated in the usual way. The mean values of these ratios and their SD were multiplied by 40 to obtain the percentage of absorption of $\mathrm{Fe}$ that corresponds to a $40 \%$ reference-dose absorption $\left(\mathrm{A}_{40 \%}\right)$. Absorption values adjusted to a $40 \%$ absorption from reference doses were chosen because they correspond to the absorption expected in subjects who are borderline Fe-deficient (10).

Mean values were compared using an unpaired two-tailed $t$ test.

Chemical measurements. The iron content in the milk samples was determined by atomic absorption spectrophotometry. The samples were freeze-dried, ashed at $600^{\circ} \mathrm{C}$ for $5 \mathrm{~h}$, and dissolved in $1: 1: 1 \mathrm{HCl} / \mathrm{HNO}_{3} / \mathrm{H}_{2} \mathrm{O}$ before analysis (11). Calcium in the milk samples was also determined by atomic absorption spectrophotometry from the same samples.

Materials. The two kinds of milk studied were 1) pooled, pasteurized, frozen human milk from a hospital milk bank (Östra Sjukhuset, Göteborg, Sweden) and 2) pasteurized, homogenized cow's milk containing $3 \%$ fat. The frozen human milk was slowly thawed in a refrigerator before use. Study 1 was made in 1984 and study 2 in 1988. Two different pools of frozen human milk were therefore used in the two studies.

The contents of calcium and iron in the samples of milk used are given in Table 2 .

\section{RESULTS}

Iron absorption from the milk samples is given in Table 1. In study 1, a significantly lower fraction of the radioiron-labeled iron in cow's milk was absorbed compared with the iron in human milk. The iron absorption ratio of cow's milk to human milk, which is the best measure of the relative iron absorption from cow's milk and human milk iron, was $0.39 \pm 0.18$ (mean $\pm \mathrm{SD})$. This value is significantly different from $1(t=10.1 ; p<$ 0.0001 ). The iron absorption from human milk, adjusted to a $40 \%$ reference-dose absorption, was $48.0 \pm 25.5 \%$ (mean $\pm \mathrm{SD}$ ). For cow's milk, the corresponding values were $19.5 \pm 17.3 \%$.

In the second study, the iron absorption from human milk, calculated in the same way, was $39.1 \pm 17.3 \%$ (mean \pm SD). This value is not statistically significantly different from the average iron absorption from human milk (48.0\%) in study 1. Addition of calcium reduced the iron absorption to $21.3 \pm$ $10.6 \%$. The mean \pm SD of the individual absorption ratio values (with and without added calcium) was $0.58 \pm 0.12$. This value is statistically significantly different from $1(t=10.1 ; p<0.0001)$, implying that calcium added to human milk has a significant inhibiting effect on iron absorption.

\section{DISCUSSION}

The present finding that the absorption of iron from human milk is significantly higher than that from cow's milk is in accordance with the results of the original comparative study (2). The relative fractional iron absorption from human milk versus cow's milk was 2.17 in the previous study and 2.46 in the present study. Adjusting the present absorption values to the individual iron status using the absorption of iron from the reference dose absorption $(40 \%)$ as a basis, the iron absorption in the present study was $19.5 \%$ from cow's milk and $48.0 \%$ from human milk. This latter iron absorption is thus of the same magnitude as reported (4) for human milk in a study on infants (49\%).

It has been speculated that human milk might contain a factor that enhances iron absorption and that would explain the higher iron absorption compared with the absorption from cow's milk. 
Table 2. Contents of iron and calcium in different batches of milk used in study 1 and study 2*

\begin{tabular}{lccccc}
\hline & \multicolumn{3}{c}{ Iron } & & \multicolumn{2}{c}{ Calcium } \\
\cline { 2 - 4 } & $\mu \mathrm{mol} / 100 \mathrm{~mL}$ & $\mathrm{mg} / 100 \mathrm{~mL}$ & & $\mathrm{mmol} / 100 \mathrm{~mL}$ & $\mathrm{mg} / 100 \mathrm{~mL}$ \\
\hline Human milk (study 1) & 0.8 & 0.045 & 2.4 & 19 \\
Cow's milk (study 1) & 0.8 & 0.045 & 0.48 & 96 \\
Human milk (study 2) & 0.7 & 0.039 & 19 & 0.48 \\
\hline
\end{tabular}

* Each serving comprised $200 \mathrm{~mL}$.

There are two possibilities to explain the difference in iron absorption between human milk and cow's milk: 1 ) the iron absorption from human milk is unexpectedly high, or 2) the iron absorption from cow's milk is unreasonably low. It is well established that the fractional iron absorption is related to iron dose and that this relationship is best described by a log dose/log absorption function (for a review, see Ref. 12). Figure 1 shows the relationship between iron absorption and the amount of iron given from simple iron solutions and human milk. The graph is based on the iron absorption from human milk in the two present studies and from two simple iron solutions containing 0.01 and $3 \mathrm{mg}$ ferrous iron in a previous study (13) on 10 subjects. The absorption from these solutions was $91.7 \pm 12.2$ and $40 \pm 7.6 \%$, respectively.

All absorption values are adjusted to the same iron status, which corresponds to an absorption of $40 \%$ from the reference dose containing $3 \mathrm{mg}$ iron. The absorption from this $3-\mathrm{mg}$ dose is one of the points in Figure 1. It is evident that the iron absorption from the two samples of human milk is much lower in relation to their iron content than the absorption from iron solutions. The present simple comparison does not support the possibility that iron in human milk is exceptionally well absorbed or that human milk contains any factor that significantly promotes the absorption of its iron. The reason for the difference in bioavailability of iron between human milk and cow's milk is thus rather that iron absorption for some reason is further reduced in cow's milk. This is not a question of only theoretical interest. It is important to clarify the cause of this lower absorption of iron from cow's milk to be able to design optimal formulas for infant nutrition based on cow's milk.

The addition of calcium chloride to human milk to equate its calcium content to that of cow's milk reduced the iron absorption from human milk by $42 \%$. However, the iron absorption from human milk with added calcium was still somewhat higher than that from cow's milk. In study 1 , the iron absorption from cow's

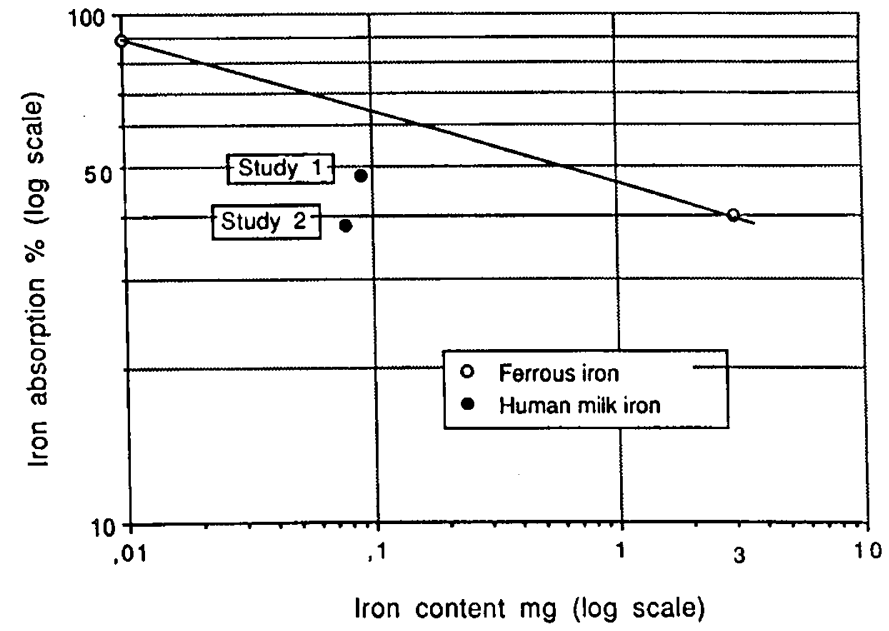

Fig. 1. Iron absorption in relation to iron content from human milk $(200 \mathrm{~mL}$ ) in study 1 and study 2 and from solutions of ferrous sulfate with different iron content. Iron absorption from the milk in the two studies is lower than expected based on their iron content only, assuming a straight line log dose/log absorption relationship (see text). milk was $19.5 \pm 17.3 \%$ (mean $\pm \mathrm{SD}$ ), and the iron absorption from human milk with added calcium in study 2 was $21.3 \pm$ $10.6 \%$. The small difference in mean values between these two kinds of milk, $2.2 \%$, was not statistically significant.

A more valid comparison of the results obtained in study 1 and study 2 can be made by comparing absorption ratios instead of means. The accuracy and precision of absorption ratios are much higher than single absorption means $\left(\mathrm{A}_{40 \%}\right.$ values), inasmuch as interindividual sources of variation in iron absorption are eliminated when each person is his own control. Using an unpaired two-tailed $t$ test $(t=2.634 ; d f 16 ; p=0.018)$, the two mean absorption ratios in the two studies ( 0.39 and 0.58$)$ were found to be statistically significantly different. This implies that the difference in calcium content between human milk and cow's milk would not fully explain the observed difference in bioavailability. The iron absorption from cow's milk was $61 \%$ lower than that from human milk (study 1 ). The reduction in iron absorption caused by adding calcium to human milk was $42 \%$ (study 2 ). Thus, the difference in calcium content would explain about $70 \%(42 / 61=68.9 \%)$ of the difference in bioavailability of iron between human milk and cow's milk. It should be mentioned that in a previous study we found that calcium in the form of calcium chloride, milk, or cheese seems to inhibit iron absorption to the same extent (6).

What is the cause of the remaining $30 \%$ difference in bioavailability between human milk and cow's milk, which is not explained by the difference in calcium content? In both present and previous absorption studies, the milk iron was extrinsically labeled with radioiron. The validity of this method of measuring iron absorption is founded on a uniform labeling of all milk iron compounds by a complete isotopic exchange between the radioiron tracer and the native milk iron compounds. Iron is present in different chemical forms in the three major chemical milk fractions: fat, casein, and whey (for a review, see Ref. 1). In the fat fraction, a major proportion of the iron is present in xanthine oxidase in the outer fat membrane; in the casein fraction, iron is associated with phosphoserine groups; and in the whey fraction it is both bound to lactoferrin and present as a low molecular weight compound. These fractions are different in size in human milk and in cow's milk. In human milk, $33 \%$ of the iron is found in the fat fraction, $9 \%$ in the casein fraction, and $58 \%$ in the whey fraction. In cow's milk, the corresponding values are 14, 24 , and $62 \%(1,11,14-16)$. A radioiron tracer added to cow's milk in vitro was found to be evenly distributed among iron in the different fractions, whereas most of the radioiron added to human milk was only found in the lactoferrin fraction (17). This distribution in human milk was also observed after peptic digestion (1). Recently, it was observed that the addition of ${ }^{59} \mathrm{Fe}$ to human milk required up to $72 \mathrm{~h}$ to reach a similar distribution among the whey, casein, and fat fractions as the intrinsic iron (18). Even if knowledge is incomplete regarding the isotopic exchange between the tracer and native iron compounds in milk during the digestion of milk, these careful biochemical and experimental studies suggest that some of the iron compounds in human milk may not be uniformly labeled by the usual extrinsic tracer methodology. One possibility would be that the much greater iron fraction in fat in human milk in the form of xanthine oxidase may not be uniformly labeled or perhaps not at all labeled by the extrinsic radioiron tracer. If not all iron in milk is labeled, the specific radioactivity of the other exchange- 
able/absorbable iron compounds in human milk will be proportionally greater, leading in turn to an overestimation of the iron absorption from human milk.

Theoretically, errors of this kind may be valid for iron absorption measurements from all kinds of foods using the extrinsic tracer. Fortunately, results with intrinsically radioiron-labeled foods indicate that this error can be disregarded (19). The extremely low iron content in the different milk fractions, however, makes this possible systematic error of incomplete isotopic exchange much more important for a food like milk than for other foods with a much higher iron content.

Two observations suggest that the determination of iron absorption from cow's milk using the extrinsic tracer gives a valid measure for iron absorption. One observation is the abovementioned even distribution in vitro of the tracer to the different chemical fractions in cow's milk (17). Another is the observation that the absorption from biosynthetically radioiron-labeled cow's milk in 10 infants was about the same as that in five infants in the same study who were given cow's milk extrinsically labeled with radioiron. These results are not conclusive because the studies were made in different infants. They still strongly suggest, however, that the extrinsic tag method is valid, inasmuch as the interindividual variation in iron absorption was rather small. It should be mentioned that this is the only study in which iron absorption from biosynthetically labeled cow's milk has been measured (5).

The difference in calcium content between human milk and cow's milk thus seems to be the main factor, explaining at least $70 \%$ of the difference in iron absorption (see above). It is reasonable to assume that a considerable part of the remaining $30 \%$ difference in iron absorption between human milk and cow's milk is explicable by an inhomogeneous labeling of iron in different milks, especially in human milk. The remaining difference might thus be considered to be an artifact, allowing the practical conclusion that the main difference in true iron absorption between human milk and cow's milk is related to their different calcium content.

The calcium content in breast milk substitutes-infant formulas-is often two to three times higher than that in human milk. A moderate excess of calcium has not been considered to have any negative effects, but has probably been used as a safety measure. Of practical importance for infant nutrition, however, is the present observation that more consideration should be devoted to the content of both calcium and iron in all such formulas (especially in the design of formulas for premature infants, because of their very high iron requirements) to ensure an optimal absorption of both elements in infants of all ages.

\section{REFERENCES}

1. Lönnerdal B 1984 Iron and breast milk. In: Stekel A (ed) Iron Nutrition in Infancy and Childhood, Nestle Nutrition Workshop Series, Vol 4. Raven Press, New York, pp 95-114

2. McMillan JA, Landaw SA, Oski FA 1976 Iron sufficiency in breast-fed infants and the availability of iron from human milk. Pediatrics 58:686-691

3. Oski FA, Landaw SA 1980 Inhibition of iron absorption from human milk by baby food. Am J Dis Child 134:459-460

4. Saarinen UM, Siimes MA, Dallman PR 1977 Iron absorption in infants: high bioavailability of breast milk iron as indicated by the extrinsic tag method of iron absorption and by the concentration of serum ferritin. $J$ Pediatr 91:36-39

5. Schulz J, Smith NJ 1958 A quantitative study on the absorption of food iron in infants and children. Am $\mathrm{J}$ Dis Child 95:109-119

6. Hallberg L, Brune M, Erlandsson M, Sandberg A-S, Rossander-Hultén L 1991 Calcium: effect of different amounts on nonheme- and heme-iron absorption in man. Am J Clin Nutr 53:112-119

7. Eakins JD, Brown DA 1966 An improved method for the simultaneous determination of iron-55 and iron-59 in blood by liquid scintillation counting. Int J Appl Radiat Isot 17:391-397

8. Björn-Rasmussen E, Hallberg L, Magnusson B, Rossander L, Svanberg B, Arvidsson B 1976 Measurement of iron absorption from composite meals. Am J Clin Nutr 29:772-778

9. Hallberg L 1980 Food iron absorption. In: Methods in Hematology. Cook JD (ed) Churchill, London, pp 116-133

10. Magnusson B, Björn-Rasmussen E, Hallberg L, Rossander L 1981 Iron absorption in relation to iron status. Model proposed to express results of food iron absorption measurements. Scand J Haematol 27:201-208

11. Fransson G-B, Lönnerdal B 1980 Iron in human milk. J Pediatr 96:380-384

12. Bothwell TH, Finch CA 1962 Iron Metabolism. Little, Brown and Co, Boston

13. Rossander-Hulthèn L, Brune M, Sandström B, Lönnerdal B, Hallberg L 1991 Competitive inhibition of iron absorption by manganese and zinc in humans. Am J Clin Nutr 54:152-156

14. Fransson G-B, Lönnerdal B 1984 Iron, copper, zinc, calcium and magnesium in human milk fat. Am J Clin Nutr 39:185-189

15. Hegenauer J, Saltman P, Ludwig D, Ripley L, Ley A 1979 Iron supplemented cow milk. Identification and spectral properties of iron bound to casein micelles. J Agric Food Chem 27:1294-1301

16. Lönnerdal B, Forsum E, Hambraeus L 1976 A longitudinal study of the protein, nitrogen and lactose contents of human milk from Swedish wellnourished mothers. Am J Clin Nutr 29:1127-1133

17. Fransson G-B, Lönnerdal B 1983 Distribution of added ${ }^{59} \mathrm{Fe}$ among different fractions of human and cow's milk. Nutr Res 3:108-109

18. Davidsson LA, Litov RE, Lönnerdal B 1990 Iron retention from lactoferrinsupplemented formulas in infant rhesus monkeys. Pediatr Res 27:176-180

19. Hallberg L 1981 Bioavailability of dietary iron in man. Annu Rev Nutr 1:123147 